\title{
Telecardiology in the Time of Corona: Study About 112 Patients
}

J.Nguadi $^{1 *}$, M.Bennani ${ }^{1}$, A.Maliki ${ }^{1}$, A.Benelmekki ${ }^{1}$, J.Kheyi ${ }^{1}$, H.Bouzelmat ${ }^{1}$, A.Chaib ${ }^{1}$, B.Mesmoudi ${ }^{2}$, R.Jennane ${ }^{2}$, Z.Mouhib $^{2}$, N.Loudiyi ${ }^{2}$, N. Mouine $^{2}$, J.Fagouri ${ }^{3}$, A.Bouchaala ${ }^{3}$, M. Boumaaz ${ }^{3}$, I.Asfalou ${ }^{3}$, M.Bennacer ${ }^{4}$, Z.Merraki ${ }^{4}$, D.Britel ${ }^{4}$, Z.Lahlafi $^{4}$, Z.Lakhal $^{4}$ and Aatif Benyass ${ }^{5}$

${ }^{1}$ Rythmology department, ${ }^{2}$ Clinical cardiology department, ${ }^{3}$ Non invasives explorations department, ${ }^{4}$ Intensive care unit, ${ }^{5}$ Chief of heart center-Rabat, MOHAMMED V UNIVERSITY OF RABAT

DOI: $10.36347 /$ sjams.2022.v10i01.004

| Received: 02.11.2021 | Accepted: 05.12.2021 | Published: 07.01.2022

*Corresponding author: J. Nguadi

Abstract

Original Research Article

The heart patients represent an unfavorable field for coronavirus disease 19 (COVID-19), and therefore, any regular follow-up consultation, not urgent, represents a great danger to catch up with this fatal disease. According to this study, we propose telemedicine as a comfortable, safe and efficient way to follow up our cardiac patients remotely.

Keywords: Telemedicine- heart failure- Covid 19- social networks.

Copyright (C) 2022 The Author(s): This is an open-access article distributed under the terms of the Creative Commons Attribution 4.0 International License (CC BY-NC 4.0) which permits unrestricted use, distribution, and reproduction in any medium for non-commercial use provided the original author and source are credited.

\section{INTRODUCTION}

The current circumstances of COVID 19 disease make elective consultation a risk factor for getting the infection, especially since the heart population represents a poor prognosis for COVID infection. However, a regular monitoring for cardiac pathologies is mandatory. We proposed a remote follow-up by telemedicine.

The Telemedicine is defined by the practice of medicine remotely using means of information and communications technology. Given the importance of clinical examination, cardiology is not very well suited to telemedicine. However, like in all medical specialities, telemedicine facilitates and supplements clinical medicine.

\section{Materials And Methods}

We report a descriptive study including 112 heart failure patients with impaired ejection fraction followed by telemedicine and social network messaging. The sex ratio of our patients is $1.1(\mathrm{M} / \mathrm{F})$ and the average age is 66.4 years.

Among our patients with impaired ejection fraction, $45 \%$ have an ischemic origin, $29 \%$ have valvular cardiopathy, $2 \%$ have hypertensive cardiopathy, $2 \%$ have left ventricular non-compaction cardiomyopathy, and $1 \%$ have post myocarditis dilated cardiomyopathy.

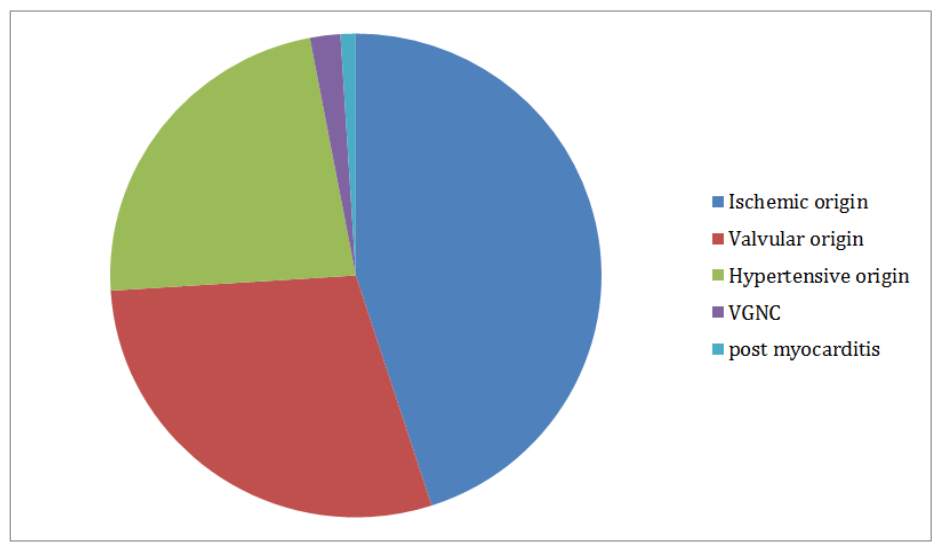

Fig-1: Etiologies of heart failure of our patients 


\section{RESULTS}

After 45 days of confinement, we analyze the consultations received and the effectiveness of the treatment based on the clinical improvement of our patients. We received 404 consultations over 45 days for us 112 patients, 24\% (98) of the consultations involved questions regarding COVID and the risk of taking the treatment, $10 \%$ (41) of the consultations were related to Ramadan and the risk of youth for our cardiac patients and $66 \%$ (265) of the consultations.

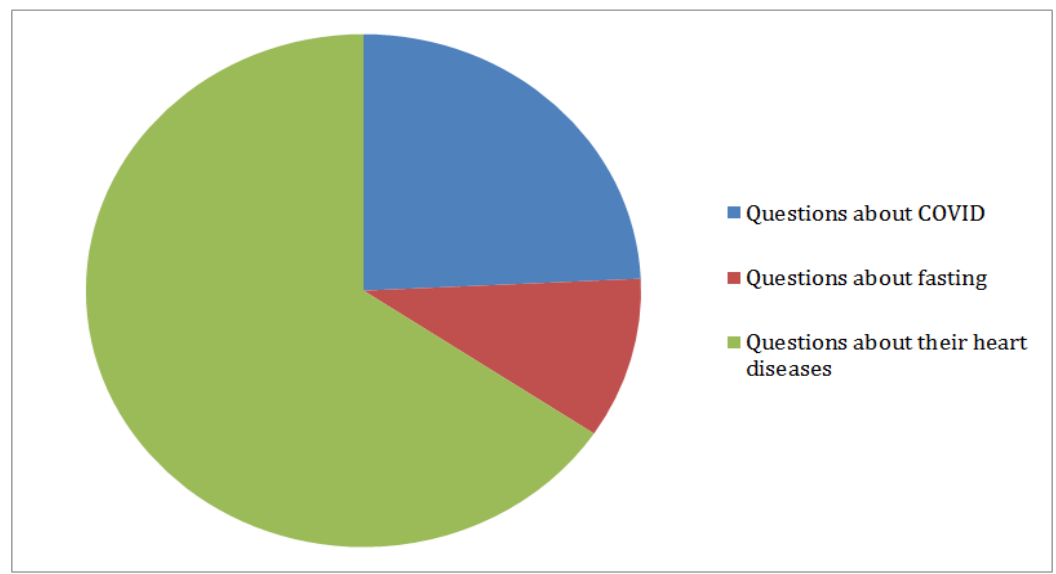

Fig-2: Consultations by telemedicine

Concerning consultations for their heart disease, $18 \%$ of our patients required a therapeutic adjustment; $30 \%$ needed to reinforce their hygienodietary measures, $12 \%$ of our patients needed a simple psychological support for psychosomatic manifestations, $38 \%$ of our patients remained asymptomatic under the same treatments and the same diet, yet 2 patients $(1.78 \%)$ required a consultation at the hospital. No case of Covid has been recorded among our study population so far.

\section{DISCUSSION}

A recent italian study has shown that the hospitalized patients with concomitant cardiac disease and COVID-19 have an extremely poor prognosis compared with subjects without a history of cardiac disease, with higher mortality, thrombo-embolic events, and septic shock rates [1]. In their review on follow-up by telephone or telemonitoring of heart failure (HF) patients, Inglis and al, show that telemedicine has an impact on all-cause mortality, with a significant reduction of $34 \%$ (p: 0.0001). This study showed a reduction in re-hospitalizations for $\mathrm{HF}$ of $20 \%$, an improvement in the quality of life of patients, costs of care, and a good acceptability of the system[2,3].

In the meta-analysis of Anker and al., studies were analyzed as part of a comparison between the effects of telemonitoring compared to usual care (noninvasive telemedicine). In this work, telemonitoring showed the reduction of the following elements: allcause mortality $(10.4 \%$ versus $15.4 \%$; $\mathrm{p}=0.001)$, admission to the hospital for all causes $(47.2 \%$ versus $52.1 \% ; \mathrm{p}=0.02)$, hospitalization linked to chronic HF (22.4\% versus $28.5 \%$; $p=0.008)[4,5]$.
Even by using simple means, telemedicine has shown its potential contribution in terms of mortality, morbidity, and in number of avoided hospitalizations. Its impact in terms of health economics is stated[6,7].

A close monitoring of patients improves the prognosis of chronic diseases and ensures the proper use of chronic treatments [7]. Through the experience of our study, we may be witnessing the birth of the «tomorrow's medicine». Indeed, in the field of chronic diseases, in terms of their epidemiology and the predictable deficit of time and resources, we need to monitor and educate better. Telemedicine based on social networks and messaging platforms is more comfortable for the treating physician than telephone calls.

\section{Conclusion}

Covid 19 revealed the value of computerized health systems and the importance of telemedicine, which even by simple means, represents an alternative to classical medicine especially in situations where the patient's movement represents a danger for him.

\section{REFERENCE}

1. Inciardi, R. M., Adamo, M., Lupi, L., Cani, D. S., Di Pasquale, M., Tomasoni, D., ... \& Metra, M. (2020). Characteristics and outcomes of patients hospitalized for COVID-19 and cardiac disease in Northern Italy. European heart journal, 41(19), 1821-1829.

2. Rosen, D., McCall, J. D., \& Primack, B. A. (2017). Telehealth protocol to prevent readmission among high-risk patients with congestive heart failure. The American journal of medicine, 130(11), 1326-1330. 
3. Burdese, E., Testa, M., Raucci, P., Ferreri, C., Giovannini, G., Lombardo, E., ... \& Feola, M. (2018). Usefulness of a telemedicine program in refractory older congestive heart failure patients. Diseases, 6(1), 10.

4. Feltner, C., Jones, C. D., Cené, C. W., Zheng, Z. J., Sueta, C. A., Coker-Schwimmer, E. J., ... \& Jonas, D. E. (2014). Transitional care interventions to prevent readmissions for persons with heart failure: a systematic review and meta-analysis. Annals of internal medicine, 160(11), 774-784.

5. Martínez-González, N. A., Berchtold, P., Ullman, K., Busato, A., \& Egger, M. (2014). Integrated care programmes for adults with chronic conditions: a meta-review. International Journal for Quality in Health Care, 26(5), 561-570.

6. Achelrod, D. (2014). Policy expectations and reality of telemedicine-a critical analysis of health care outcomes, costs and acceptance for congestive heart failure. Journal of telemedicine and telecare, 20(4), 192-200.

7. Pandor, A., Thokala, P., Gomersall, T., Baalbaki, H., Stevens, J. W., Wang, J., ... \& Fitzgerald, P. (2013). Home telemonitoring or structured telephone support programmes after recent discharge in patients with heart failure: systematic review and economic evaluation. Health Technology Assessment (Winchester, England), 17(32). 